\title{
NTRK3 gene fusion in an adult ganglioglioma: illustrative case
}

\author{
Sebastian Rubino, MD, ${ }^{1}$ John Lynes, MD, ${ }^{1}$ Paul McBride, MD, ${ }^{1}$ Solmaz Sahebjam, MD, ${ }^{1}$ Sepideh Mokhtari, MD, ${ }^{1}$ \\ Joaquim M. Farinhas, MD, ${ }^{2}$ Arie Perry, MD, ${ }^{4}$ Robert Macaulay, MD, ${ }^{3}$ and Michael A. Vogelbaum, MD, $\mathrm{PhD}{ }^{1}$ \\ Departments of ${ }^{1}$ Neuro-Oncology, ${ }^{2}$ Radiology, and ${ }^{3}$ Pathology, Moffitt Cancer Center, Tampa, Florida; and ${ }^{4}$ Department of Pathology, University of California, San Francisco, \\ California
}

\begin{abstract}
BACKGROUND Gangliogliomas are well-differentiated, slow-growing glioneuronal neoplasms frequently reported to harbor upregulating alterations in the mitogen-activated protein kinase pathway, particularly serine-threonine protein kinase B-RAF alterations. Fusions involving neurotrophin tyrosine receptor kinase (NTRK) genes have rarely been reported in ganglioglioma. Similarly, echinoderm microtubule-associated protein-like (EML) 4 gene fusion has been described in lung cancer, but none has been reported in ganglioglioma.

OBSERVATIONS This report discusses the care of a 72-year-old man presenting with medication-refractory, left-sided focal seizures who was found to have a nongadolinium-enhancing, T2-hyperintense, right frontoparietal lesion. The patient received resection, and histological analysis found a World Health Organization grade I ganglioglioma, with genetic analysis demonstrating an EML4-NTRK3 gene fusion protein.

LESSONS To our knowledge, this is the first report of an NTRK3 fusion, EML4-NTRK3, in an adult ganglioglioma, which is otherwise mostly associated with BRAF alterations and activation of the mitogen-activated protein kinase signaling pathway. Further studies are needed to elucidate the function of the resultant fusion protein and determine whether it may serve as a future therapeutic target.
\end{abstract}

https://thejns.org/doi/abs/10.3171/CASE21645

KEYWORDS ganglioglioma; neurotrophin tyrosine receptor kinase; NTRK; EML4-NTRK3; gene fusion

Gangliogliomas have been described in the literature as early as $1947^{1}$ and are classically described as well-differentiated, slow-growing glioneuronal neoplasms composed of dysplastic ganglion cells and neoplastic glial cells. They are the most common epilepsy-associated neoplasm and account for $2 \%$ of all primary tumors. ${ }^{2}$ Although they are typically histologically low grade, anaplastic transformation of the glial component can rarely occur. ${ }^{3}$ They can present at any age, although most are diagnosed in children and young adults before 30 years of age, with a slight male preponderance. ${ }^{4}$ They vary radiographically, histologically, and geographically within the central nervous system (CNS), including supratentorial, infratentorial, and spinal intramedullary lesions. Lobar gangliogliomas disproportionately present with seizures rather than focal neurological deficit or elevated intracranial pressure. These lesions are histologically defined by dysmorphic ganglion cells intermixed with neoplastic glial cells of either astrocytic (most common) or oligodendroglial (rare) morphology and can contain eosinophilic granular bodies, Rosenthal fibers, calcifications, and perivascular lymphocytes. Challenges in histological diagnosis have led to further investigation via genetic profiling of these tumors. Pekmezci et al. investigated 40 histologically determined gangliogliomas from 1990 to 2017 and found that $90 \%$ of these tumors harbored mutations that upregulate the mitogen-activated protein (MAP) kinase pathway, with $68 \%$ of tumors having serine-threonine protein kinase B-RAF (BRAF) alterations. ${ }^{2}$ However, this study did not report any fusions involving neurotrophin tropomyosin receptor kinase (NTRK) 1, 2, or 3.

Fusions involving NTRK genes have been investigated in gliomas, affecting $\leq 2 \%$ of gliomas with varying tumor histology among different age groups. Infantile NTRK-fused gliomas have been shown to have predominately high-grade histology, pediatric NTRKfused gliomas typically involve NTRK2 and range from low to high

ABBREVIATIONS CNS = central nervous system; EML = echinoderm microtubule-associated protein-like; MAP = mitogen-activated protein; $\mathrm{MRI}=$ magnetic resonance imaging; NTRK = neurotrophin tyrosine receptor kinase; PI3-K = phosphatidylinositol-3-kinase; PLC- $\gamma=$ phospholipase $\mathrm{C}-\mathrm{gamma}$; TRK = tropomyosin receptor kinase; WHO = World Health Organization

INCLUDE WHEN CITING Published January 31, 2022; DOI: 10.3171/CASE21645.

SUBMITTED November 11, 2021. ACCEPTED December 13, 2021.

(C) 2022 The authors, CC BY-NC-ND 4.0 (http://creativecommons.org/licenses/by-nc-nd/4.0/). 
histology, and adult NTRK-fused gliomas typically involved NTRK1 and had predominantly high-grade histology. ${ }^{5}$ An EML4-NTRK3 fusion was previously reported in a glioma cell line, ${ }^{6}$ and only one case of an NTRK fusion, TLE4-NTRK2, has previously been reported in an adult ganglioglioma. ${ }^{7}$ Two NTRK2 gene fusions were also recently reported in pediatric gangliogliomas (one fused to KCTD16 and one to TRIM24). ${ }^{8}$ To the authors' knowledge, this is the first report of an NTRK3 fusion, EML4-NTRK3, in an adult ganglioglioma, further expanding the known genetic profile of these CNS tumors.

\section{Illustrative Case}

A 72-year-old right-handed man with hypertension, hyperlipidemia, diabetes mellitus type 2, squamous cell skin cancer, bladder cancer, chronic sinusitis, and prior transient ischemic attack was referred to Moffitt Cancer Center for neurological and neurosurgical evaluation of recalcitrant focal seizures. The patient had developed changes in vision and loss of balance approximately 6 months prior to this evaluation. At that time, head computed tomography and brain MRI studies revealed a right frontal lesion appearing to be radiologically most consistent with a low-grade glioma. Outside neurosurgical evaluation elected observation with 6-month radiographic follow-up due to a concern for the risk of surgical morbidity. Thereafter, the patient began to experience intermittent numbness along his left face, arm and leg that was thought to be focal seizures with postictal left hemibody weakness and headaches. He was started on antiepileptic medication by an outside neurologist with progressive increase in dosage and number of agents, with decreased seizure frequency but continued breakthrough episodes. He presented to the authors' institution for second surgical opinion after follow-up MRI showed a $3.6 \times 3.3 \times$
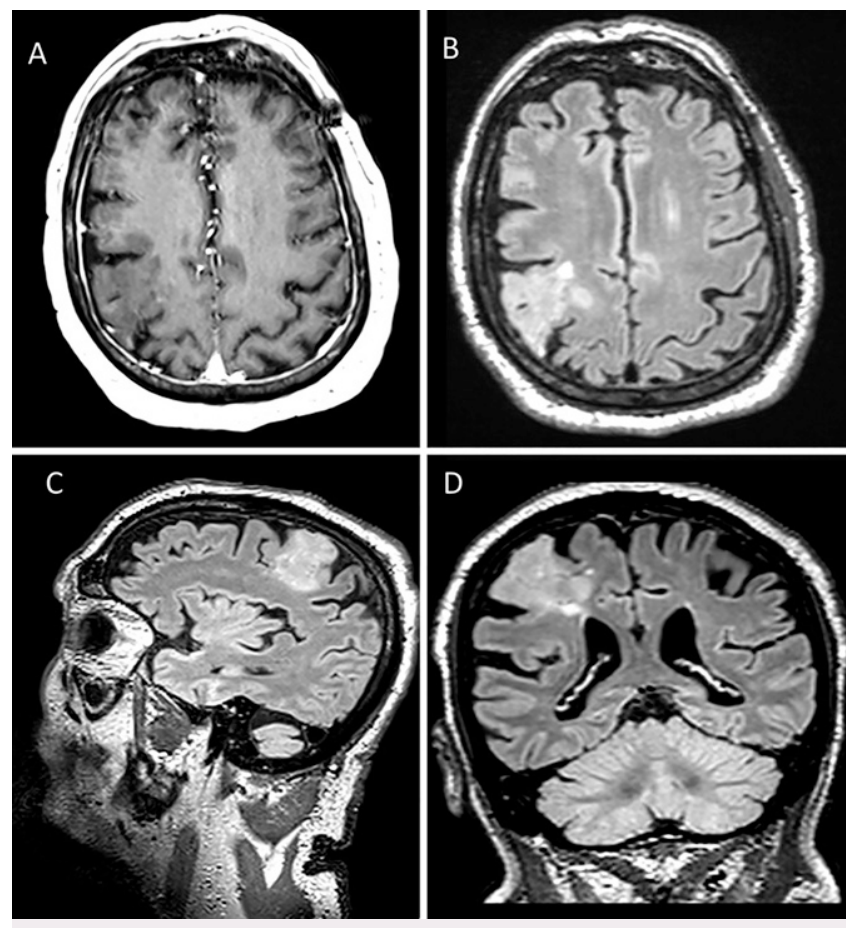

FIG. 1. Preoperative MRI studies. A: T1-weighted postgadolinium axial image demonstrating no pathologic enhancement. Axial (B), sagittal (C), and coronal (D) T2-weighted fluid-attenuated inversion recovery images. 3.2-cm expansile cortical lesion in the right frontoparietal region. The lesion was stable in size, included small cystic components, and demonstrated increased T2-weighted fluid-attenuated inversion recovery signal without enhancement (Fig. 1). The patient underwent awake right frontal craniotomy for resection of the lesion with electrocorticography, direct stimulation of motor areas, and intraoperative monitoring of motor/sensory function.

Pathologic evaluation demonstrated mildly atypical glial cells intermixed with dysmorphic ganglion cells consistent with a ganglioglioma, World Health Organization (WHO) grade I (Fig. 2). There was no mitotic activity, microvascular proliferation, or necrosis. Immunostaining was positive for GFAP, OLIG2, and CD34 in hypercellular zones. The tumor cells were negative for $p 53$, mutant IDH1 $\mathrm{R} 132 \mathrm{H}$, and BRAF V600E mutant protein. The Ki-67 labeling index was low $(<1 \%)$. Genetic analysis with the UCSF500 next-generation sequencing cancer gene panel demonstrated an EML4-NTRK3 fusion. The patient recovered well postoperatively with resolution of headaches and seizures and improvement in left-sided strength and apraxia. He is being managed with serial imaging evaluations.

\section{Discussion}

The anticipated 2021 WHO classification of tumors of the CNS continues to characterize gangliogliomas under the heterogenous group of "glioneuronal and neuronal tumors," with known molecular

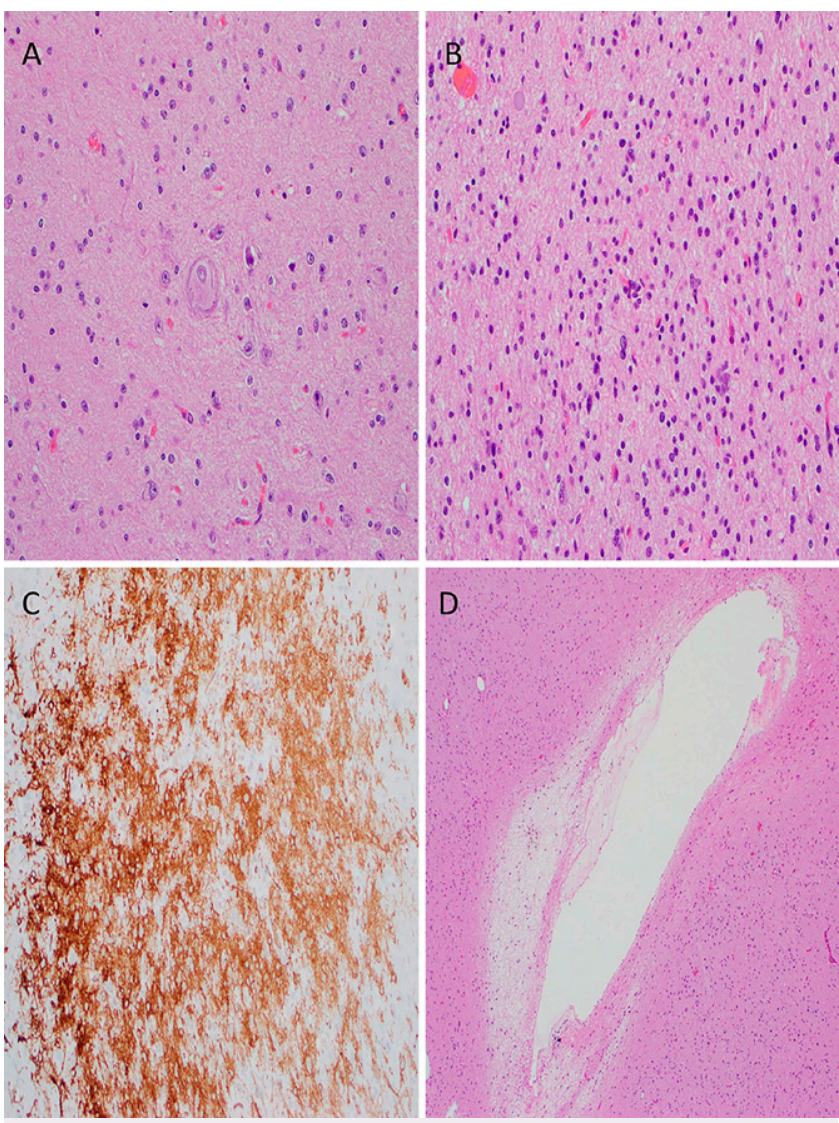

FIG. 2. Histological sections of the tumor with representative features. A: Dystrophic neuron (H\&E stain, original magnification $\times 200$ ). B: Hypercellularity $(H \& E$ stain, original magnification $\times 200)$. C: $C D 34+$ cell populations (immunohistochemistry stain, original magnification $\times 200)$. D: Microcavitations (H\&E stain, original magnification $\times 100)$. 
alterations in BRAF for ganglion cell tumors and various other signature copy number and gene alterations associated with other glioneuronal tumor types. ${ }^{9}$ Herein, we report the first NTRK3 fusion, EML4-NTRK3, in an adult ganglioglioma. As additional molecular signatures become known, it becomes essential for providers to develop and maintain an understanding of the molecular signaling pathways to understand how these genetic alterations and their downstream effectors may be related and how potential novel therapeutics alter these signaling pathways.

\section{The TRK Family}

NTRK genes (NTRK 1, 2, and 3) encode tropomyosin receptor kinase (TRK) proteins, including TRKA, TRKB, and TRKC proteins, which are membrane-bound proteins that dimerize, phosphorylate, and activate downstream signaling cascades via phospholipase C-gamma (PLC- $\gamma$ ), phosphatidylinositol-3-kinase/protein kinase B (PI3K/AKT), MAP kinase, and protein kinase $C$ pathways when bound with neurotrophins; ultimately, this leads to activation of downstream effectors responsible for neuronal survival and differentiation. $^{10,11}$ TRK receptors are highly expressed in CNS tissue and have been shown to play a physiological role in neuronal development, proliferation, survival, and synaptic plasticity. ${ }^{12}$ TRK activation in cancer has been identified to occur via mutations, splice variants, TRK overexpression, and, most commonly, NTRK fusions. ${ }^{11}$ NTRK fusions typically occur when the $3^{\prime}$ sequences of NTRK1, NTRK2, or NTRK3 (including kinase domain) are juxtaposed to the $5^{\prime}$ sequences of a different gene, with most fusion partners harboring oligodimerization domains. The exact mechanism of oncogenic activation is unknown; however, the two predominant theories include (1) the gene partner replacing an autoinhibitory domain and subsequently allowing for unrestricted downstream activation of the constitutively active $3^{\prime}$ sequence and/or (2) the gene partner actively promoting and perpetuating downstream cellular activation. Although initially identified in colorectal and papillary carcinomas, NTRK fusions have been found in multiple tumor types, including reports of NTRK $1^{13}$ and NTRK2 fusions ${ }^{7}$ in gangliogliomas and other glioneuronal tumors. ${ }^{14}$ Epigenetic regulation via methylation of normal NRTK genes has been reported as correlating with increasing grade of glioma samples compared to control tissue. ${ }^{15}$ A wide range of NTRK gene fusions has been reported in various low- and high-grade adult CNS malignancies. ${ }^{16}$

\section{The Echinoderm Microtubule-Associated Protein-Like Family}

Humans express a family of six echinoderm microtubule-associated protein-like (EML) proteins thought to contribute to the regulation of microtubule assembly during mitosis. ${ }^{17} E M L 1$ to EML4 have an N-terminal coiled-coil domain, followed by a C-terminal domain containing a hydrophobic EML protein domain and variable tryptophan-aspartic acid repeats; ${ }^{18}$ these oligomerization domains make these proteins prime NTRK fusion partners. EML4 was involved in the first oncogenic fusion discovered in lung cancer as part of an EML4-ALK (anaplastic lymphoma kinase) fusion identified in nonsmall-cell lung cancer, ${ }^{19}$ and this fusion protein has been extensively studied in non-small-cell lung cancer and found to have downstream effects via the PLC- $\gamma$, PI3K, Ras, and JAK3 pathways. $^{20-23}$ Interestingly, the EML4-ALK fusion proteins have both similar properties shared with their parent proteins, such as microtubule binding and trimerization and kinase activity, as well as unique properties, such as HSP90 dependence. ${ }^{17}$ However, the question "Does EML4 fusion simply remove kinase inhibition, or does it intrinsically contribute to pathway activation?" remains to be answered.

\section{Observations}

To our knowledge, this is the first report of an NTRK3 fusion, EML4-NTRK3, in an adult ganglioglioma, which is otherwise mostly associated with BRAF alterations and activation of the MAP kinase signaling pathway. Although potentially still activating this pathway, the NTRK pathway can activate downstream signaling cascades via PLC- $\gamma$, PI3K/protein kinase $B$, and protein kinase $C$ pathways. Further investigation is needed to better understand how this fusion participates in oncogenesis. Elucidating the various unique activities of this fusion protein may help to develop novel therapeutics against tumors expressing this abnormal protein with less off-target downstream effects rather than using inhibitors that target ubiquitously expressed TRK proteins.

\section{Lessons}

NTRK3 fusion, EML4-NTRK3, is a newly reported gene fusion in an adult ganglioglioma. Further studies are needed to elucidate the function of the resultant fusion protein and determine whether it may serve as a future therapeutic target.

\section{References}

1. Bailey $P$, Beiser H. Concerning gangliogliomas of the brain. J Neuropathol Exp Neurol. 1947;6(1):24-34.

2. Pekmezci M, Villanueva-Meyer JE, Goode B, et al. The genetic landscape of ganglioglioma. Acta Neuropathol Commun. 2018;6(1):47.

3. Gatto L, Franceschi E, Nunno VD, Tomasello C, Bartolini S, Brandes AA. Glioneuronal tumors: clinicopathological findings and treatment options. Future Neurol. 2020;15(3):FNL47.

4. Im SH, Chung CK, Cho BK, et al. Intracranial ganglioglioma: preoperative characteristics and oncologic outcome after surgery. $J \mathrm{Neu}$ rooncol. 2002;59(2):173-183.

5. Torre M, Vasudevaraja V, Serrano J, et al. Molecular and clinicopathologic features of gliomas harboring NTRK fusions. Acta Neuropathol Commun. 2020;8(1):107.

6. Klijn C, Durinck S, Stawiski EW, et al. A comprehensive transcriptional portrait of human cancer cell lines. Nat Biotechnol. 2015;33(3):306-312.

7. Prabhakaran N, Guzman MA, Navalkele P, Chow-Maneval E, Batanian JR. Novel TLE4-NTRK2 fusion in a ganglioglioma identified by array-CGH and confirmed by NGS: potential for a gene targeted therapy. Neuropathology. Published online March 4, 2018. doi: 10.1111/neup.12458.

8. Zhao X, Kotch C, Fox E, et al. NTRK fusions identified in pediatric tumors: the frequency, fusion partners, and clinical outcome. JCO Precis Oncol. 2021;1:PO.20.00250.

9. Louis DN, Perry A, Wesseling P, et al. The 2021 WHO classification of tumors of the central nervous system: a summary. Neuro Oncol. 2021;23(8):1231-1251.

10. Khotskaya YB, Holla VR, Farago AF, Mills Shaw KR, MericBernstam F, Hong DS. Targeting TRK family proteins in cancer. Pharmacol Ther. 2017;173:58-66.

11. Cocco E, Scaltriti M, Drilon A. NTRK fusion-positive cancers and TRK inhibitor therapy. Nat Rev Clin Oncol. 2018;15(12):731-747.

12. Amatu A, Sartore-Bianchi A, Bencardino K, Pizzutilo E, Tosi F, Siena S. Tropomyosin receptor kinase (TRK) biology and the role of NTRK gene fusions in cancer. Ann Oncol. 2019;30(suppl 8):viii5-viii15.

13. Kurozumi K, Nakano $Y$, Ishida J, et al. High-grade glioneuronal tumor with an ARHGEF2-NTRK1 fusion gene. Brain Tumor Pathol. 2019;36(3):121-128. 
14. Deng MY, Sill M, Chiang J, et al. Molecularly defined diffuse leptomeningeal glioneuronal tumor (DLGNT) comprises two subgroups with distinct clinical and genetic features. Acta Neuropathol. 2018;136(2):239-253.

15. Palani M, Arunkumar R, Vanisree AJ. Methylation and expression patterns of tropomyosin-related kinase genes in different grades of glioma. Neuromolecular Med. 2014;16(3):529-539.

16. Gambella A, Senetta R, Collemi G, et al. NTRK fusions in central nervous system tumors: a rare, but worthy target. Int J Mol Sci. 2020;21(3):E753.

17. Sabir SR, Yeoh S, Jackson G, Bayliss R. EML4-ALK variants: biological and molecular properties, and the implications for patients. Cancers (Basel). 2017;9(9):E118.

18. Eichenmuller B, Everley P, Palange J, Lepley D, Suprenant KA. The human EMAP-like protein-70 (ELP70) is a microtubule destabilizer that localizes to the mitotic apparatus. J Biol Chem. 2002;277(2):1301-1309.

19. Soda M, Choi YL, Enomoto M, et al. Identification of the transforming EML4-ALK fusion gene in non-small-cell lung cancer. Nature. 2007;448(7153):561-566.

20. Reungwetwattana T, Weroha SJ, Molina JR. Oncogenic pathways, molecularly targeted therapies, and highlighted clinical trials in nonsmall-cell lung cancer (NSCLC). Clin Lung Cancer. 2012; 13(4):252-266.

21. Roskoski R Jr. Anaplastic lymphoma kinase (ALK): structure, oncogenic activation, and pharmacological inhibition. Pharmacol Res. 2013;68(1):68-94.
22. Solomon B, Wilner KD, Shaw AT. Current status of targeted therapy for anaplastic lymphoma kinase-rearranged non-small cell lung cancer. Clin Pharmacol Ther. 2014;95(1):15-23.

23. Raghav KPS, Gonzalez-Angulo AM, Blumenschein GR Jr. Role of HGF/MET axis in resistance of lung cancer to contemporary management. Transl Lung Cancer Res. 2012;1(3):179-193.

\section{Disclosures}

Dr. Sahebjam reported research fundings from Merck, Bristol Myers Squibb, and Brooklyne ImmunoTherapeutics as well as personal fees from Boehringer Ingelheim outside the submitted work. No other disclosures were reported.

\section{Author Contributions}

Conception and design: Vogelbaum, Rubino, Lynes, Perry, Macaulay. Acquisition of data: Vogelbaum, Rubino, Lynes, Perry, Macaulay. Analysis and interpretation of data: Vogelbaum, Rubino, Lynes, Sahebjam, Farinhas, Perry, Macaulay. Drafting the article: Rubino, Lynes. Critically revising the article: all authors. Reviewed submitted version of manuscript: Vogelbaum, Rubino, Lynes, McBride, Sahebjam, Farinhas, Perry, Macaulay. Approved the final version of the manuscript on behalf of all authors: Vogelbaum. Administrative/technical/material support: Macaulay. Study supervision: Vogelbaum.

\section{Correspondence}

Michael A. Vogelbaum: Moffitt Cancer Center, Tampa, FL. michael. vogelbaum@moffitt.org. 